\title{
Biofunctional catheter coatings based on chitosan-fatty acids derivatives
}

\author{
Agata Niemczyk ${ }^{\mathrm{a}}$, Agata Goszczyńska ${ }^{\mathrm{a}}$, Monika Gołda-Cępa ${ }^{\mathrm{b}}$, Andrzej Kotarba ${ }^{\mathrm{b}}$, \\ Peter Sobolewski ${ }^{\mathrm{a}}$, Miroslawa El Fray ${ }^{\mathrm{a}, *}$ \\ ${ }^{a}$ Division of Functional Materials and Biomaterials, Faculty of Chemical Technology and Engineering, West Pomeranian University of Technology, Szczecin, Al. Piastow 45, \\ 71-311, Szczecin, Poland \\ ${ }^{\mathrm{b}}$ Faculty of Chemistry, Jagiellonian University, Gronostajowa 2, 30-387, Kraków, Poland
}

A R T I C L E IN F O

\section{Keywords:}

Coatings

Surface modification

XPS

Chitosan

Fatty acid

Microbial adhesion

\begin{abstract}
A B S T R A C T
Multifunctional and biofunctional coatings for medical devices are an attractive strategy towards tailoring the interactions of the device with the body, thereby influencing the host response, and the susceptibility to microbial colonization. Here we describe the development of a coating process to yield amphiphilic, lubricious coatings, resistant to bacterial colonization, based on chitosan. Chitosan-fatty acid derivatives were obtained by simultaneous $\mathrm{N}, \mathrm{O}$-acylation of chitosan with either linoleic, $\alpha$-linolenic, or dilinoleic acid. Chemical characterization of new materials was carried out using ${ }^{1} \mathrm{H}$ NMR, FTIR, and XPS. Surface properties of coated polyester samples were studied using SEM and contact angle measurements, which indicated that the incorporation of hydrophobic constituents into chitosan macromolecules led to a decrease of both surface roughness and water contact angle. Importantly, tribological testing demonstrated that these new coatings decrease the coefficient of friction due to the self-organization of fatty acid (from 0.53 for the neat chitosan to 0.35 for chitosan-fatty acid derivative). Meanwhile, preliminary bacterial colonization tests indicated significant-over $80 \%$-reduction in $E$. coli colonization following coating with chitosan-linoleic and chitosan- $\alpha$-linolenic derivatives. Finally, cytotoxicity and hemocompatibility studies confirmed that all amphiphilic chitosan-fatty acid derivatives were non-toxic and non-hemolytic. Collectively, our results demonstrate the potential of the developed coating strategy, particularly the chitosan-linoleic and chitosan- $\alpha$-linolenic acid derivatives, for applications as biofunctional catheter coatings.
\end{abstract}

\section{Introduction}

The invention of catheters and development of catheterization procedures have been extremely significant milestones in the history of the medicine. While having their origins in the urological field (Feneley, Hopley, \& Wells, 2015), these medical devices are now ubiquitous, including intravascular applications, such as providing vascular access (Lin \& Wu, 2018), as well as a growing range of endovascular diagnostic and interventional applications in cardiology (Canfield \& Totary-Jain, 2018) and radiology (Baum \& Baum, 2014). A great deal of recent research has focused on modification of the surfaces (Ikada, 1994) of these devices, with a major focus on hydrophilic coatings (Wyman, 2012) using a range of polymers, such as silicone, polyvinyl alcohol (PVA), polyvinyl pyrrolidone (PVP), and polyethylene glycol (PEG). The primary aim has been improving the wetting and lubricity of the devices, but also patient comfort. However, in the case of cardiac catheterization, mechanical, friction-induced damage of the blood vessel lumen may be a major cause of complications
(Dellimore, Franklin, \& Helyer, 2014; Sobolewski \& El Fray, 2015). Meanwhile, antifouling/antimicrobial properties have also been of great interest, particularly for urological applications (Nacey \& Delahunt, 1991; Singha, Locklin, \& Handa, 2017), but also for intravascular use (Casey, Mermel, Nightingale, \& Elliott, 2008). Moreover, existing coatings are not without their problems, particularly in the intra- and endovascular fields: flaking or detachment of the coating from the device can cause adverse effects ranging from granuloma (Chopra et al., 2017; Gilchrist \& Kozak, 2010; Mehta \& Mehta, 2018) to potentially fatal embolism (Chopra et al., 2017; Mehta \& Mehta, 2018). The unique complexity of the interactions at the tissue-material interface during a catheterization procedure motivates further research into coating development, particularly multifunctional or biofunctional coatings. At present, among diverse lubricious coatings dedicated to medical applications, there are only few examples of the coatings exhibiting multi/biofunctionality (Parada, Yuk, Liu, Hsieh, \& Zhao, 2017). From a materials point of view, amphiphilic chitosan derivatives (Aranaz, Harris, \& Heras, 2010) are particularly promising for coating

\footnotetext{
* Corresponding author.

E-mail addresses: aniemczyk@zut.edu.pl (A. Niemczyk), mirfray@zut.edu.pl (M. El Fray).
} 
development, thanks to the antimicrobial potential and biodegradability of chitosan. Our group has been focused on chitosan-fatty acid derivatives, due to their unique physico-chemical properties (Niemczyk, Kaczorowski, \& El Fray, 2015; Niemczyk, Kmieciak, El Fray, \& Piegat, 2016), including antimicrobial activity (Niemczyk \& El Fray, 2013). Importantly, we recently demonstrated for the first time the potential of chitosan-derivatives for improving lubricity (Niemczyk, El Fray, \& Franklin, 2015).

Of course, in addition to the choice of coating material, the coating application procedure is a crucial aspect of coating development, particular in light of the recent focus on adverse effects associated with coating flaking or delamination (Chopra et al., 2017; Mehta \& Mehta, 2018). The surface chemistry of commonly used medical device polymers, such as polyamides and polyurethanes, represents a major challenge, due to hydrophobicity (surface free energies in the range of $20-40 \mathrm{mN} / \mathrm{m}$ ) and very low surface functionality (or lack of thereof). These two intrinsic features hinder wettability of the surface with a coating solution and limit, or even preclude, the chemical bonding between the coating and surface (Theapsak, Watthanaphanit, \& Rujiravanit, 2012). Here our goal was to develop a coating procedure for covalently bonding amphiphilic chitosan-fatty acid derivatives (CHFA) to poly(aliphatic/aromatic-ester) substrate, a biomedically-relevant class of polymers (El Fray \& Czugala, 2012). First, we implemented surface plasma treatment, combined with oxygen flushing, to activate the surface. This is an easy-to-apply procedure for a diverse range of polymeric materials, that provides high efficiency of functional group formation, without influencing bulk properties of the material and can be incorporated in an industrial production line (Ikada, 1994; Lee, Hsiue, \& Kao, 1996). Next, we utilized carbodiimide coupling chemistry to covalently link CHFA to the surface and obtain coatings. We hypothesized that this combination of chitosan derivatives and coating strategy would yield stable multifunctional coatings, because the plasma-treatment would result in a highly hydrophilic PET-DLA substrate surface capable of attracting the hydrophilic chitosan chains of the CHFA derivatives, while repelling long, hydrophobic FA chains to the outer part of the coating. Further, the biomacromolecules would have the ability to reorganize-a key feature in terms of influencing functional properties, in particular lubricity and antimicrobial properties. To explore these aspects, we conducted detailed wetting analysis, using static and dynamic contact angle measurements, as well as X-ray photoelectron spectroscopy depth elemental analysis, in order to describe the behavior and organization of the biomacromolecules forming the coatings. The obtained coatings were non-toxic and demonstrated improved lubricity and resistance to bacterial colonization, while preserving hemocompatibility.

\section{Experimental Section}

\subsection{Synthesis procedure}

Chitosan-fatty acid derivatives (CHFA) were obtained by chemical modification of chitosan (CH) (ChitoClear ${ }^{\circledR} 43000$ - hqg10, Primex ehf Iceland Company, Siglufjordur, Iceland; $\mathrm{DD}=82 \% \quad-{ }^{1} \mathrm{H}$ NMR, $\mathrm{M}_{\mathrm{n}}=20 \mathrm{kDa}, \mathrm{M}_{\mathrm{w}}=146 \mathrm{kDa}$ - GF C-H PLC (SI)) with three different fatty acids (FA): $\alpha$-linolenic (ALA), linoleic (LA) (Sigma-Aldrich, Darmstadt, Germany) or dilinoleic acid (DLA) (Pripol 1009, molecular weight $\sim 570 \mathrm{~g} / \mathrm{mol}$, Croda, The Netherlands). For each FA, the synthesis was carried out as described previously (Niemczyk et al., 2016). Briefly, fatty acid (ALA, LA, or DLA) and 1-ethyl-3-(3-dimethylaminopropyl)carbodiimide (EDC) coupling agent (Sigma-Aldrich, Darmstadt, Germany) $(1: 1 \mathrm{~mol} / \mathrm{mol})$ were dissolved in methanol and stirred for $3 \mathrm{~h}$ (for ALA and LA at room temperature, for DLA at $60^{\circ} \mathrm{C}$ ). Next, the reaction mixture was added dropwise to a $1 \mathrm{wt} \%$ chitosan solution in a mixture of $1 \%$ of acetic acid and methanol $(2: 1 \mathrm{v} / \mathrm{v}))$. The reaction of FA with chitosan was carried out at a pH between 5 and 6 for $24 \mathrm{~h}$, at room temperature for ALA and LA or $60^{\circ} \mathrm{C}$ for DLA. The weight ratio of each FA to chitosan used in the synthesis was fixed at 0.17 . The final product was precipitated with methanol/ammonia solution $(7: 3 \mathrm{v} / \mathrm{v})$, centrifuged, washed with distilled water and methanol, and dried for $48 \mathrm{~h}$ under vacuum at $60^{\circ} \mathrm{C}$.

\subsection{Substrate material}

Multiblock thermoplastic elastomer consisting of poly(ethylene terephthalate) (PET) hard segments and butylene dilinoleate (DLA) soft segments in a 50:50 wt.\% ratio was used as the substrate material for chitosan-derivative coatings. This copolymer (Shore D 53) is part of a series of PET-DLA thermoplastic elastomers, ranging from rubber-like to rigid (Piegat \& El Fray, 2007), developed and extensively studied in our group for various biomedical applications, including blood-contacting devices, such as heart assist devices (El Fray \& Czugala, 2012; Staniszewski et al., 2017; Staniszewski, Sobolewski, Piegat, \& El Fray, 2018). The kilo-scale synthesis of PET-DLA copolymer, including twostep polymerization (transesterification and polycondensation from the melt) has been described in detail previously (Piegat \& El Fray, 2007). Here, test samples (Figs. S1, SI) were obtained by injection molding using BOY 35A injection molding machine (BOY Machines, Germany). The barrel temperature range was $200-210{ }^{\circ} \mathrm{C}$ and the mold temperature was $20^{\circ} \mathrm{C}$. The injection and holding pressure were 890 and 793 bar, respectively. Samples in the form of discs (Fig. S1A) were used for all tests, except for the tribological tests, where hemispherical probes (Fig. S1B) were used. Before biological tests, samples were washed with deionized water, dried, and then sterilized with ethylene oxide at $30{ }^{\circ} \mathrm{C}$. After the sterilization process, samples were incubated in an aerator for $2 \mathrm{~h}$.

\subsection{Coating process}

CHFA-coatings on PET-DLA substrate were applied via 3-step dipcoating procedure. First, PET-DLA samples were treated with argon plasma (Nano Plasma system with RF Generator from Diener Electronic, Ebhausen, Germany), in order to enrich the PET-DLA substrate surface with functional groups, yielding hydrophilic character of the surface and improving surface wetting with the film-forming solution. The plasma activation process involved: (1) providing argon into the chamber ( $2 \mathrm{~min})$, (2) activating plasma at pressure of $0.25 \mathrm{bar}(30 \mathrm{~min})$, and (3) flushing the chamber with pure oxygen ( $2 \mathrm{~min}$ ). Next, the newly formed peroxide and carboxylic groups on the PET-DLA substrate were activated using the carbodiimide coupling agent, EDC, by immersing of the plasma-treated samples in an aqueous EDC solution $(5 \mathrm{~g} / \mathrm{L})$ at room temperature for $3 \mathrm{~h}$. Next, the samples were dipped into the CHFA coating solution for $24 \mathrm{~h}$, to allow covalent bonds to form between carboxylic groups of the substrate and the amino and/or hydroxyl groups of the coating material. The coating solution was prepared by dissolving CHFA (2 $\mathrm{wt} \%)$ in a $2: 1(\mathrm{v} / \mathrm{v})$ mixture of $1 \%$ of acetic acid and methanol. Subsequently, the samples were rinsed with diluted acetic acid and water to remove unbound CHFA layer, then with diluted basic solution $(0.1 \mathrm{M} \mathrm{NaOH})$ to saponify the surface by removing acetate moieties, and finally with water. Samples were subsequently dried for $24 \mathrm{~h}$ under vacuum at $40^{\circ} \mathrm{C}$.

\subsection{Nuclear magnetic resonance (NMR) spectroscopy}

${ }^{1} \mathrm{H}$ NMR spectroscopic measurements were performed using a Bruker DPX 400 Avance III HD spectrometer, operating at $400.2 \mathrm{MHz}$. Approximately $5 \mathrm{mg}$ of chitosan and CHFA derivatives was dissolved in $500 \mu \mathrm{L}$ of solvent mixture $(2: 8 \mathrm{v} / \mathrm{v})$ of $1 \%$ acetic acid-d 4 in deuterium oxide (v/v; Sigma-Aldrich, Darmstadt, Germany) and methanol-d4 (Deutero GMBH, Kastellaun, Germany). DSS (4,4-dimethyl-4-silapentane-1-sulfonic acid, ARMAR Chemicals, Dottingen, Switzerland) was used as an internal chemical shift standard. The 1D diffusion-filtered ${ }^{1} \mathrm{H}$ NMR spectra were acquired using $1 \mathrm{D}$ stimulated spin-echo 
pulse sequence with bipolar gradients and LED (ledbpgp2s1d) ( $\mathrm{Wu}$, Chen, \& Johnson, 1995) for effective suppression of the water and methanol signals, while retaining the signals from the analyzed polymer.

\subsection{Infrared spectroscopy (IR)}

Infrared spectrometer with attenuated total reflectance module (ATR FT-IR, ALPHA, Bruker) was used to obtain infrared spectra for structural characterization of CHFA. For each sample, 32 scans were averaged across the spectral range of $400-4000 \mathrm{~cm}^{-1}$ with a resolution of $2 \mathrm{~cm}^{-1}$. Prior to analysis, samples were dried at $60{ }^{\circ} \mathrm{C}$ under vacuum for $24 \mathrm{~h}$. To evaluate the $\mathrm{N}, \mathrm{O}$-substitution of CHFA the absorption intensities of the characteristic bands i.e. amide (A), ester (B) and alkyl (C) were determined and divided by the absorption intensity of the reference band (R) (Niemczyk et al., 2016).

\subsection{X-ray photoelectron spectroscopy (XPS)}

The elemental composition of CHFA and the coatings deposited on PET-DLA substrate was determined by XPS in an ultra-high vacuum system, equipped with an SES R4000 (Gammadata, Scienta System AB, Sweden) analyzer. The monochromatic Al Ka source (1486.6 eV) operated at $350 \mathrm{~W}$ was applied. The vacuum in the spectrometer chambers was less than $5 \times 10^{-9} \mathrm{mbar}$. The acquired XPS spectra were analyzed using Casa-XPS 2.3.15 software. All spectra were calibrated using the adventitious $\mathrm{C}$ 1s peak, with a fixed value of $284.5 \mathrm{eV}$. Additionally, for depth elemental analysis of the CHALA coating, four argon ion sputtering steps were performed: the first three steps at $4 \mathrm{kV} 2 \mathrm{~mA}$ TPD $1 \mathrm{~ms}$ parameters over $10 \mathrm{~min}$ and the fourth over $30 \mathrm{~min}$.

\subsection{Scanning Electron Microscopy (SEM)}

Surface morphology of uncoated and CHFA-coated substrate was visualized with scanning electron microscopy (FE-SEM SU-70, Hitachi). Before imaging, the surfaces were gold sputtered with a $60 \mathrm{~nm}$ thick layer.

\subsection{Contact angle measurements}

Static and dynamic contact angle measurements (sessile drop shape analysis) of the uncoated and chitosan/CHFA-coated surfaces were performed using a Krüss DSA 100 Drop Shape Analyzer goniometer equipped with a camera and recording system. Static contact angle measurements were carried out using $2 \mu \mathrm{L}$ ultrapure water $\left(\theta_{\text {water }}\right)$ or dimethyl sulfoxide (DMSO, Sigma-Aldrich, Darmstadt, Germany) $\left(\theta_{\text {DMSO }}\right)$ drop placed on the surface and recorded for $30 \mathrm{~s}$. The average contact angle was calculated from at least 5 measurements and the five measurement points at $1,5,10,20,30$ th sec were selected. Dynamic contact angle measurements were conducted by the placing a $2 \mu \mathrm{L}$ drop of ultrapure water on the surface, followed by increasing the volume to $7 \mu \mathrm{L}$ (advancing contact angle, $\theta_{\mathrm{a}}$ ) and then decreasing back to $2 \mu \mathrm{L}$ (receding contact angle, $\theta_{\mathrm{r}}$ ), at a flow rate $0.33 \mu \mathrm{L} / \mathrm{s}$. The average dynamic contact angle was also calculated from at least 5 measurements.

\subsection{Tribological properties - coefficient of friction}

The coefficient of friction $(\mu)$ of the uncoated and chitosan/CHFAcoated samples was determined using a reciprocating sliding friction test, conducted using a previously developed biotribological system (Niemczyk, El Fray et al., 2015) intended to model the conditions occurring during an endovascular catheterization procedure. Briefly, the tests were carried out using a CETR UMT Multi-Specimen Test System (Brïker, USA) with a total sliding distance of $0.9 \mathrm{~m}$ using a PET-DLA probe (Fig. S1 B) rubbed against a fixed, flat piece of soft poly(vinyl alcohol) hydrogel (PVA, Elvanol 90-50, DuPont) immersed in glycerol/ water solution ( $44 \mathrm{wt} . \%$ ). The test parameters: load, velocity, and stroke were $0.1 \mathrm{~N}, 0.5 \mathrm{~mm} / \mathrm{s}$, and $10 \mathrm{~mm}$, respectively. Each test was repeated at least five times using a fresh sample for each test. The results are presented as line graphs showing the change in $\mu$ over time (1800s) and as an average value $\left(\mu_{\mathrm{av}}\right)$ calculated from the $\mu$ values $(n=5)$ obtained over the measurement range from 800 to 1700 s.

\subsection{Bacterial colonization tests}

The resistance to bacterial colonization of uncoated and CHFAcoated samples was evaluated by assessing adhesion of Gram-negative bacteria, Escherichia coli (ATCC-25922, E. coli), to the surfaces. The bacterial suspension ( $40 \mu \mathrm{l}$, PBS buffer, $0.5 \mathrm{McF}$ arland) was placed onto the surface of a sample disc, incubated for $15 \mathrm{~h}$, and then flushed using phosphate buffered solution (PBS) to rinse off non-adhered bacterial cells. The adhered cells were then removed from surface by intensive shaking in a saponin solution $(0.2 \%$ in PBS) for 20 min. Next, serial 10fold dilutions (from $10^{-1}$ to $10^{-4}$ ) were prepared and used to inoculate $(100 \mu \mathrm{L})$ brain heart infusion agar (BHIA, Merck KGaA, Darmstadt, Germany) solid media for the colony forming unit (CFU) assay. Following $24 \mathrm{~h}$ of incubation, any bacterial colonies present were counted: one colony on solid media represents one cell that had adhered to the surface of the sample. Data were analyzed using R software (RStudio), first by checking ANOVA assumptions using Bartlett's test for equal variances and Shapiro-Wilk test for normality of residuals. As both tests rejected their null hypotheses, data was analyzed using nonparametric Kruskal-Wallis test, followed by non-parametric post hoc test: "many-to-one" Conover-Iman test with Holm p-value adjustment for multiple comparisons.

\subsection{Cytotoxicity studies}

Cytotoxicity tests were performed based on the "direct contact" procedure of ISO 10993-5 (ISO, 2009). Briefly, murine fibroblasts cells (L-929, ATCC CCL-1) were cultured in Medium 199 with $10 \%$ fetal bovine serum (FBS) until 70-80\% confluence and discs of each material were placed onto the cell layers. After $24 \mathrm{~h}$ of contact, cell viability was assessed using the fluorescence microscopy AxioObserver (Carl Zeiss, Germany) and the vital dyes fluorescein diacetate (FDA, Merck KGaA, Darmstadt, Germany) and propidium iodide (PI, Merck KGaA, Darmstadt, Germany). For image acquisition and analysis AxioVision 4.8 (Carl Zeiss, Germany) software was used. The microscopic assessment included quantification of the number of necrotic cells in each visual field, as well as observation of the morphology of living cells, including adhesion and density in order to rate reactivity in accordance with ISO 10993-5, from grade 0 (nonreactive - nontoxic group) to 4 (severe toxic group). As a reference material Bionate II 55D (DSM Biomedical Inc.), further referred to as Bionate, was used.

\subsection{Hemocompatibility tests}

The hemocompatibility study was conducted using human whole blood (HWB) obtained from the Regional Blood Center in Katowice (Poland), stored in citrate-phosphate-dextrose-adenine (CPDA, Merck KGaA, Darmstadt, Germany). The samples (uncoated and coated with CHFA-derivatives) were placed in contact with HWB for $24 \mathrm{~h}$ at $37^{\circ} \mathrm{C}$. As a negative control, HWB without contact with the test materials was used. Hemocompatibility was evaluated by measuring: (i) free hemoglobin concentration in plasma (fHGB $(\mathrm{g} / \mathrm{L})$ ) using a HemoCue spectrophotometer, (ii) morphological parameters of blood and red blood cell indices, measured with a hematology analyzer, (iii) blood cell morphology, and (iv) the degree of hemolysis, which was determined by calculating the hemolytic index (HI). The gradation of $\mathrm{HI}$ was in accordance with ASTM F756-00 (ASTM, 2000): (I) HI $=0-2 \%$ not hemolytic, (II) $\mathrm{HI}=2-5 \%$ slightly hemolytic, and (III) $\mathrm{HI}>5 \%$ hemolyzing. The same Bionate material as mentioned previously was used a 

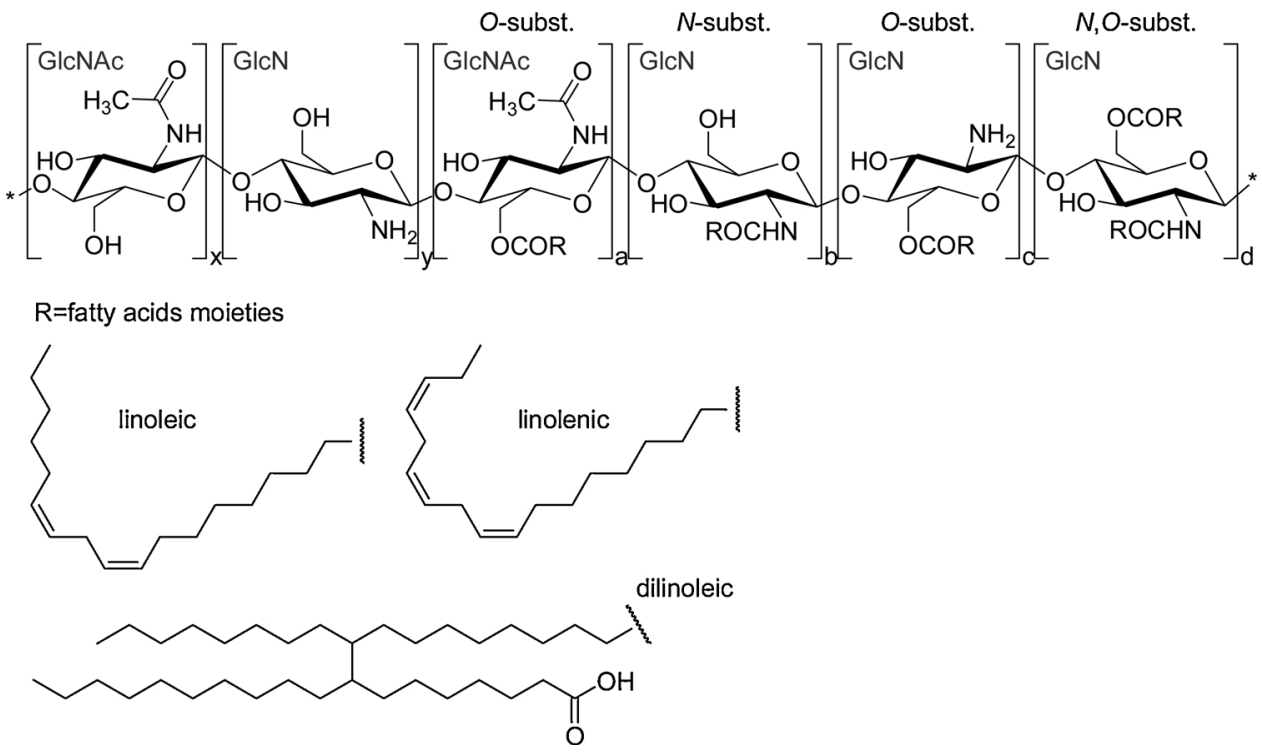

Fig. 1. Possible chemical structures of CHFA.

reference.

\section{Results and discussion}

\subsection{Chitosan derivatives}

The N,O-acylated CHFA derivatives were obtained as a result of carbodiimide coupling carried out in acidic environment. The acidic reaction medium, which led to partial protonation of amino groups, permitted the less reactive primary hydroxyl groups of chitosan to react with FA. The partial protonation also served to retain some amino groups in derivative structure, while simultaneously allowing us to avoid commonly applied additional stages of blocking and unblocking reactions with phthalic anhydride and hydrazine, respectively (Kurita, Ikeda, Shimojoh, \& Yang, 2007). As a consequence of simultaneous $N$ and $O$-acylation reactions between chitosan and FA the structure of each CHFA derivative is complex: composed of various $\mathrm{N}-, \mathrm{O}-$, and $\mathrm{N}, \mathrm{O}-$ acylated chitosan glucopiranose units, as shown in Fig. 1. This complexity of CHFA derivative structures, which can have six different glucopiranose units, requires various techniques to evaluate the effect of $\mathrm{N}$ - and $\mathrm{O}$-acylation substitution on the properties of the obtained derivative. Therefore, spectroscopic analysis using NMR, IR, and XPS was performed to provide insight into the role of CHFA structure on coating properties. The simultaneous $\mathrm{N}, \mathrm{O}$-acylation reaction approach was selected due to economic and environmental concerns, as well as the possibility of obtaining derivatives with some unreacted amino groups in the main chain, which are essential for some biological properties of chitosan, such as antimicrobial properties and biocompatibility towards human cells (Croisier \& Jérôme, 2013; Oliveira, Martins, Mafra, \& Gomes, 2012).

The chemical modification of chitosan with each of the three different fatty acids was confirmed by ${ }^{1} \mathrm{H}$ NMR spectroscopy analysis. The ${ }^{1} \mathrm{H}$ NMR spectra of the chitosan and CHFA derivatives are provided in Fig. 2. The individual spectra are presented in Supplementary Information in Figs. S2-5.

Briefly, for $\mathrm{CH}$, the signal at $2.0 \mathrm{ppm}$ and at $3.09 \mathrm{ppm}$ is assigned to the three protons of $\mathrm{N}$-acetyl group in $\mathrm{N}$-acetyl glucopiranose unit (GlcNAc) and the $\mathrm{H} 2$ proton of the aminoglucopiranose (GlcN) residue, respectively. The ring protons, $\mathrm{H} 3-\mathrm{H} 6$ of GlcN and $\mathrm{H} 2-\mathrm{H} 6$ of GlcNAc, appear as a set of overlapping signals in the range 3.4-4.0 ppm, while the anomeric proton $\mathrm{H} 1$ of GlcNAc resonates at $4.55 \mathrm{ppm}$. The appearance of the typical FA moiety signals in the NMR spectra of the chitosan derivatives confirms the successful grafting of the molecule along the chitosan backbone. The signal at approximately $2.33 \mathrm{ppm}$ in each NMR spectrum is attributed to the alpha methylene group in the FA chain, while the signals of the terminated methyl group and long methylene chain units are located between 0.8 and $1.6 \mathrm{ppm}$. Further, the small signal at $5.33 \mathrm{ppm}$ is assigned to the protons of double bound $\mathrm{HC}=\mathrm{CH}$ in LA and ALA. The changes within the glucopiranose unit signals in the spectra of CHFA are related to the substitution of both hydroxyl and amine group. In view of overlapping of the $\mathrm{H} 6$ and $\mathrm{H} 2$ signals that are attached to these functional groups, it is difficult to evaluate the exact substitution location and degree.

The FTIR spectra of chitosan and CHFA derivatives are presented in the Fig. 3. The expected $\mathrm{N}, \mathrm{O}$-acylated structure is reflected by increased intensity of the absorption band at $1641 \mathrm{~cm}^{-1}$ corresponding to $\mathrm{CO}=$ stretching vibration of amide groups ( $N$-acylated part of derivative) and by the presence of a new absorption band at $1741 \mathrm{~cm}^{-1}$ corresponding to $\mathrm{CO}=$ stretching vibration of ester groups ( $O$-acetylated part of derivative). Additionally, a significant increase in absorption is observed in the $2930-2850 \mathrm{~cm}^{-1}$ region corresponding to aliphatic chains of FA. The modification of chitosan is further reflected in absorption bands at: 3430,1250 and $1198 \mathrm{~cm}^{-1}$ as a result of $O$-acylation, 3430 and $1550 \mathrm{~cm}^{-1}$ as a result of $N$-acylation, and 1435 and $1419 \mathrm{~cm}^{-1}$ as a result of alkyl chain presence.

The absorption bands: amide (A) at $1641 \mathrm{~cm}^{-1}$, ester (B) at $1741 \mathrm{~cm}^{-1}$, and alkyl (C) at $2853 \mathrm{~cm}^{-1}$ can be considered as characteristic bands of the CHFA chemical structure. The comparison of these IR absorption bands, presented as a ratio of a characteristic band to the reference band, for unmodified chitosan and each of the derivatives provides a detailed description of the presence of these functional groups in the structure of CHFA. The Fig. S6 (SI) summarizes these absorption band ratios for chitosan and the CHFA derivatives as follows: A/R - the relative amount of amide groups corresponding to progress of $\mathrm{N}$-acylation reaction, $\mathrm{B} / \mathrm{R}$ - the relative amount of the ester groups corresponding to progress of $O$-acylation reaction, and C/R - the relative amount of alkyl groups, corresponding to the substitution degree in terms of increase in alkyl-fatty acid chain presence.

For both chitosan derivatives obtained with C18-fatty acids (LA and ALA) there are noticeable differences in the structure, namely the degree and the place of FA substitution, despite the fact that both derivatives were synthesized using the same reaction conditions. This result is consistent with our previous observations for similar chitosan derivatives synthesized with a higher amount of four different C18-fatty acids (Niemczyk et al., 2016). The presence of three double bonds in the cis conformation in ALA versus two in LA chains may affect mobility, 


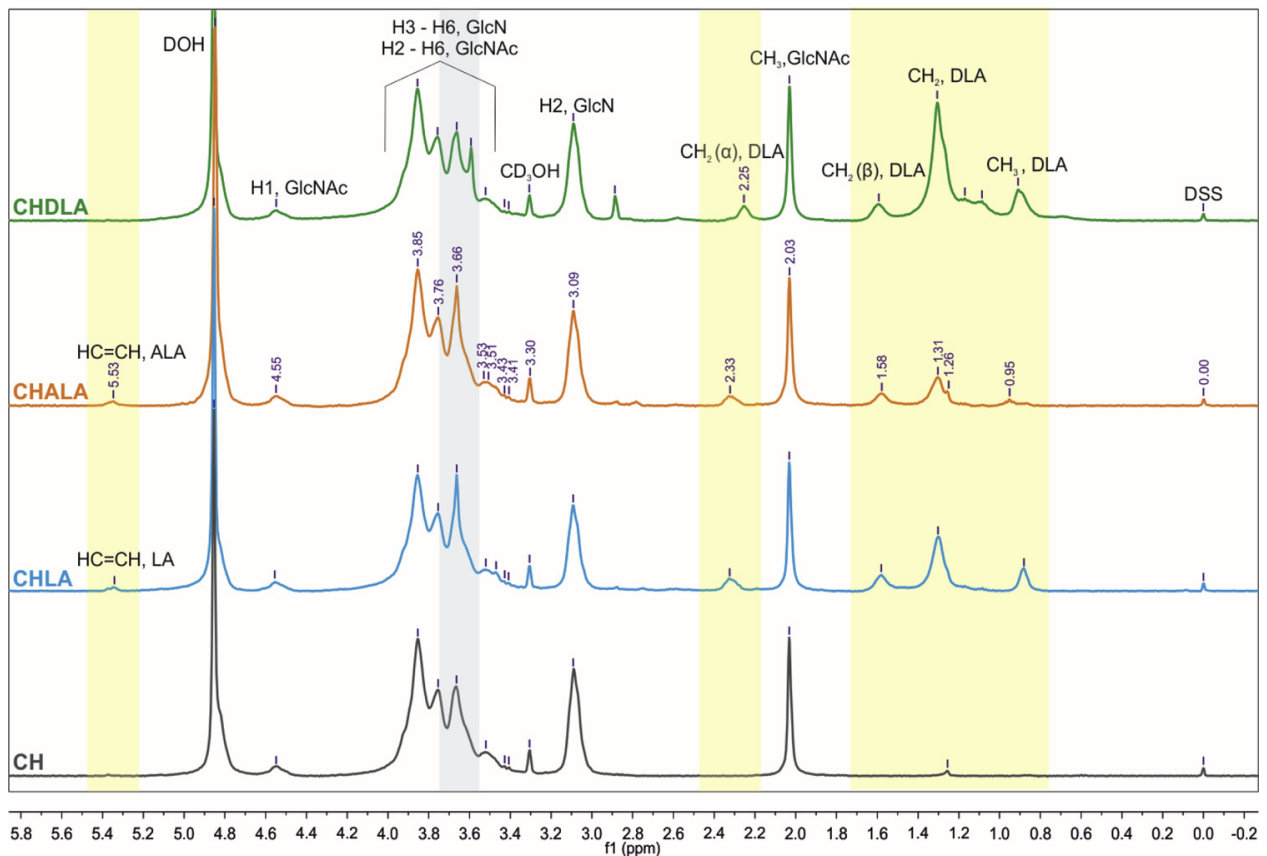

Fig. 2. The ${ }^{1} \mathrm{H}$ NMR spectra of chitosan and CHFA.

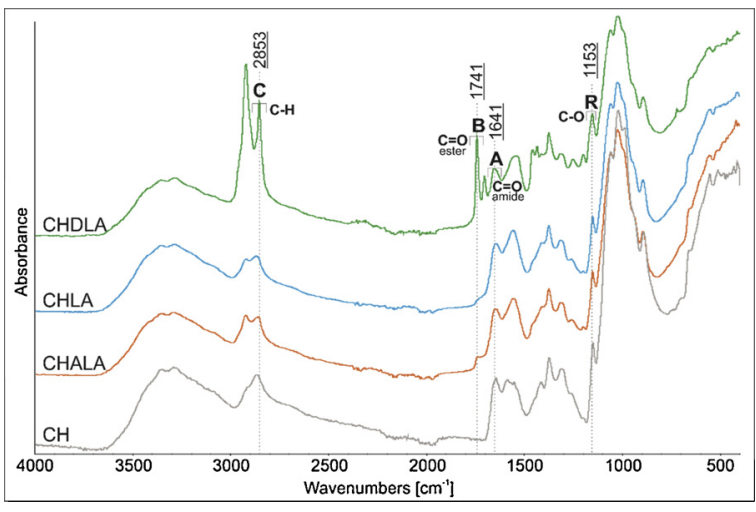

Fig. 3. ATR-FTIR spectra of chitosan and CHFA.

influencing the course of the reaction and the final derivative structure. CHALA is characterized by higher acid substitution, as more alkyl chains are present in the structure: the C/R ratio of CHALA and CHLA is 1.19 and 0.66 , respectively. Due to the fact that the CHALA derivative has a higher amount of amide groups $(A / R=1.76)$ than CHLA (A/ $\mathrm{R}=0.99$ ), while the difference in ester groups amount between derivatives is smaller, it can be assumed that the main structural difference is due to differences in the progress of the $N$-acylation reaction. For CHDLA, the high $\mathrm{C} / \mathrm{R}$ ratio indicates greatest degree of substitution, even after accounting for the fact that DLA is a C18 dimer, while the markedly increased $\mathrm{B} / \mathrm{R}$ ratio, compared to CHLA and CHALA, indicates that this is due to greater degree of $O$-acylation.

The elemental surface composition of chitosan and CHFA derivatives determined by XPS is provided in Table S1 (SI). For chitosan, the surface concentration (at.\%) of carbon, oxygen, and nitrogen were determined to be 62,31 , and 7 at.\%, respectively. The chemical modification of chitosan with long aliphatic chains resulted in changes in the atomic ratio, depending on the type of the FA and its substitution degree. Atomic C 1s concentrations for the CHLA and CHALA were similar and greater than that of $\mathrm{CH}$ by a few percent. The small change in the carbon concentration indicates low substitution degree of the derivatives, which is consistent with the analysis of IR spectra. The highest C 1s concentration, 84.8 at $\%$, was obtained for the CHDLA, confirming the highest substitution degree of this derivative.

The analysis of high resolution C 1s spectra, included in the Table S1 and presented in the Fig. S7 (SI), indicated the presence of three bands with binding energy maximum at approx. $284.5 \mathrm{eV}, 286 \mathrm{eV}$, and $288 \mathrm{eV}$ ( $289 \mathrm{eV}$ for CHDLA). The first band at $284.5 \mathrm{eV}$ is attributed to a single $\mathrm{C}-\mathrm{C}$ and $\mathrm{C}-\mathrm{H}$ bond; therefore, an increase in the intensity of this band can be correlated with substitution degree of FA. The next band at $286 \mathrm{eV}$ is characteristic for single carbon bonded to heteroatom as $\mathrm{C}-\mathrm{O}$ and $\mathrm{C}-\mathrm{N}$, corresponding to carbon bonds with hydroxyl and amino groups, as well as glycosidic bridges present in the $\mathrm{CH}$ and CHFA structures. The last band $(\sim 288 \mathrm{eV})$ is attributed to bonds of carbon with two oxygen atoms or one oxygen and nitrogen atoms, i.e. acetal, amide, ester or carboxylic functional group.

The high resolution $\mathrm{N}$ 1s spectra analysis of chitosan and CHFA revealed presence of two bands at approx. 399.8 and $401.5 \mathrm{eV}$. The first band corresponds to amine and amide groups and the second to the protonated amine groups. The presence of the protonated $-\mathrm{NH}_{3}{ }^{+}$group in chitosan, CHLA, and CHALA structures indicates that some acid molecules are ionically bound to amino groups and this is more noticeable for $\mathrm{CH}$ and CHLA derivatives-the CHDLA derivative does not have any ionically bound molecules in the structure.

When comparing the structure of the two C18 derivatives, CHLA and CHALA, it can be noticed that CHLA, which has more protonated amino groups (1.1 at.\%), has a lower $\mathrm{C} / \mathrm{O}$ atomic ratio (equal to 2.2 ), as compared to CHALA, which has values of 0.6 at.\% and 2.7, respectively. This can indicate that the ionically bound molecules are small molecules of acetic acid, rather than long FA molecules. The ionic binding of the low molecular weight acids by chitosan and its derivatives is quite common, as small acid molecules can be easily 'trapped' between the chitosan/chitosan-derivative macromolecules (Dambies, Guimon, Yiacoumi, \& Guibal, 2001). However, the presence of bonded shortchain acid is at a very low level, and therefore usually does not influence the main properties of the final product.

\subsection{Preparation and characterization of coatings}

The surface activation and dip-coating procedure was successfully applied to yield thin coatings of chitosan and CHFA derivatives covalently bound to PET-DLA substrate. To verify the change of PET-DLA 


\begin{tabular}{|c|c|c|c|c|c|c|}
\hline$\theta_{\text {water }}=95^{\circ}$ & plasma Ar & $\begin{array}{c}21^{\circ} \\
\star \star \star \star \star \star \star \star \star \star \star \star \star \star \star \star \star\end{array}$ & & $\begin{array}{l}68^{\circ} \\
\end{array}$ & & $76^{\circ}$ \\
\hline \multirow{2}{*}{$\begin{array}{l}\text { PET-DLA } \\
\text { substrate }\end{array}$} & treatment & \multirow{2}{*}{$\begin{array}{l}\text { PET-DLA } \\
\text { substrate }\end{array}$} & functional group. & \multirow{2}{*}{$\begin{array}{l}\text { PET-DLA } \\
\text { substrate }\end{array}$} & CHLA & \multirow{2}{*}{$\begin{array}{l}\text { PET-DLA } \\
\text { substrate }\end{array}$} \\
\hline & $+\mathrm{O}_{2}$ flushing & & activation & & dip-coating & \\
\hline
\end{tabular}

Fig. 4. Change in static water contact angle (measured after $5 \mathrm{~s}$ ) of the PET-DLA substrate following each step of the activation and CHFA dip-coating procedure (an example for CHLA).

surface wettability after plasma treatment and subsequent dip-coating steps, we monitored the static water contact angle $\left(\theta_{\text {water }}\right)$, as shown in Fig. 4.

The initial $\theta_{\text {water }}$ of the PET-DLA surface is approx. 95. Argon plasma treatment combined with subsequent oxygen flushing of the substrate resulted in a decrease to $21^{\circ}$, thus the surface exhibited hydrophilic properties. The hydrophilicity is a direct consequence of the newly formed peroxide and carboxylic groups on the surface. Following the next steps of the process, i.e. carboxylic groups activation and dip coating, the change in $\theta_{\text {water }}$ confirmed the successful functionalization and presence of the coating.

SEM images of uncoated and coated samples, shown in Fig. 5, revealed substantial differences in surface morphologies. Neat, uncoated PET-DLA (Fig. 5A) exhibits different crystalline structures on the surface, resulting in a rough and non-uniform topography. This is the result of the semicrystalline character of the PET-DLA copolymer and the crystalline structures that are formed during the polymer processing from the melt.

In terms of possible biomedical applications, micrometric roughness and/or surface defects are undesired, because they can cause serious health complications by increasing risk of microorganism adhesion, resulting in infection, or protein/platelet adhesion inducing inflammation or thrombosis. However, the SEM images indicate that the presence of CHFA derivative coatings on the PET-DLA surface leads to smoother and more uniform surfaces at the microscale (Fig. 5, B-D).

The presence of the coatings on the substrate surface was confirmed by XPS spectral analysis. Three basic constituting elements, i.e. carbon, oxygen and nitrogen, were identified on the surface and the atomic concentrations of all coatings are summarized in Table S2. Because the
PET-DLA copolyester consists only of carbon, oxygen, and hydrogen, the presence of nitrogen is direct evidence of $\mathrm{CH}$ or CHFA coating presence, as the only sources of nitrogen (N 1s) are the $\mathrm{CH}$ or CHFA molecules.

The amount of nitrogen detected on the coating surface ( $~ 2$ at.\%) is significantly lower than the amount previously determined for $\mathrm{CH}$ or the derivatives themselves ( $\sim 5-7$ at.\%). Some authors have attempted to correlate the amount of nitrogen with the efficiency of the coating process (Huh et al., 2001); however, the sampling depth of XPS measurements is within the range of $0.5-7.5 \mathrm{~nm}$, thus the specific conformation or organization of coating macromolecules can have a major effect (Anastasiadis, Retsos, Pispas, Hadjichristidis, \& Neophytides, 2003). From our experience, the lower nitrogen concentration is likely a result of self-assembly and the organization of the amine/amide groups toward the inside part of the coating of chitosan-derivative macromolecules, due to their amphiphilic properties. This macromolecular self-assembly and reorganization hypothesis for our CHFA derivative coatings was confirmed by additional XPS depth-profiling elemental analysis of the CHALA coating. The measurements were performed using argon ion sputtering to effectively erode the CHALA coating over $60 \mathrm{~min}$, with five XPS spectra collected after 10, 20, 30, and $60 \mathrm{~min}$ of the sputtering process. The atomic concentration of carbon, oxygen and nitrogen at each time point are summarized in the Table S3 (SI). The intensity of photoelectrons in the range of binding energy 395-405 eV, determining the concentration of nitrogen, is presented in Fig. 6.

As can be seen in Fig. 7, every subsequent ion sputtering of CHALA coating revealed an increased nitrogen concentration. The changes in $\mathrm{N}$ 1 s elemental concentration induced upon sputtering can be explained by the arrangement of the amphiphilic macromolecules within the coating, indicating a higher contribution of chitosan chains in deeper layers. As the nitrogen amount is increasing with the depth, it suggests that the FA chains are mostly located in the outer layers of the coating.

The specific spatial arrangement of chitosan or CHFA derivatives within the coating is a function of the ability of the amphiphilic macromolecules to self-assemble during the coating process-primarily during drying. We conclude that, as anticipated, the hydrophilic, plasma-treated PET-DLA surface attracts the hydrophilic chitosan
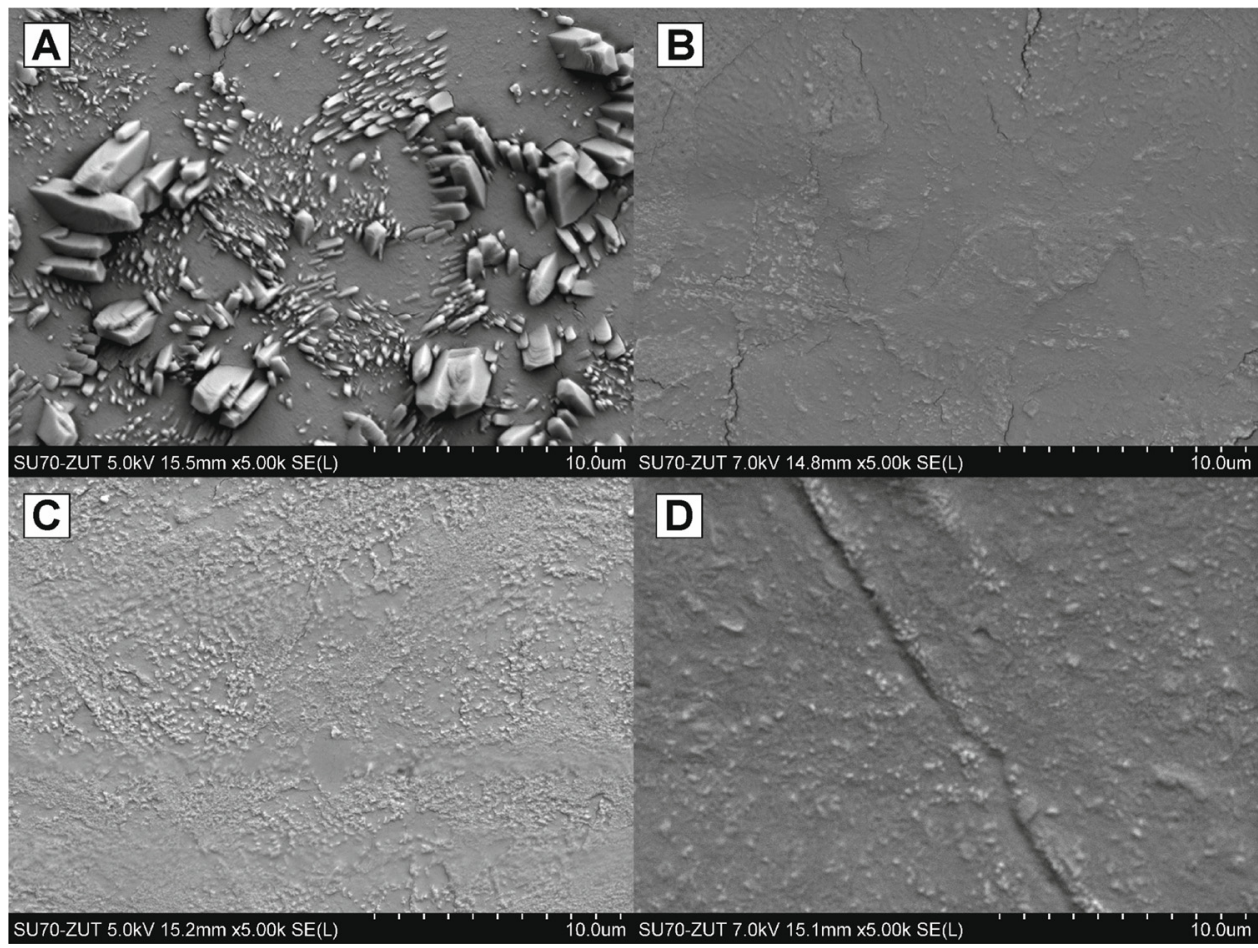

Fig. 5. SEM images of the surface morphology of the neat PET-DLA (A) and CHLA (B), CHALA (C) and CHDLA (D) coatings (5k magnification). 


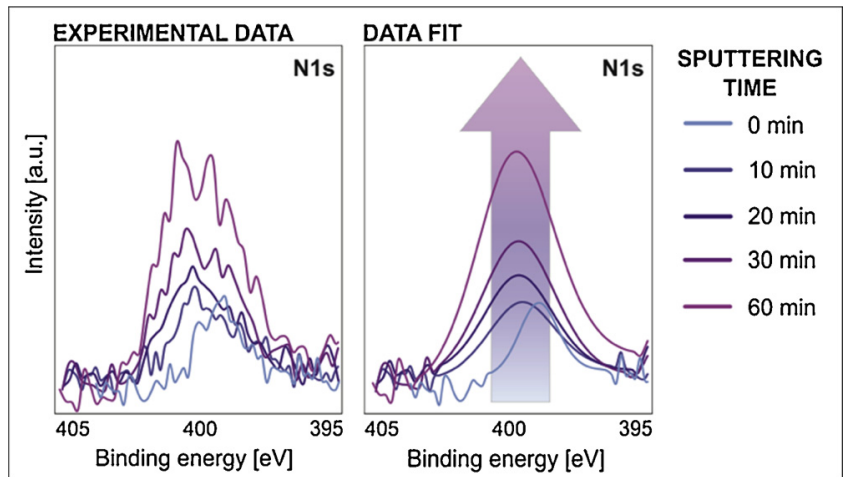

Fig. 6. Depth profiling for CHALA coating, revealing differences in nitrogen $\mathrm{N}$ 1 s concentration as a function of sputtering time, with higher $\mathrm{N}$ amount detected at greater depth.
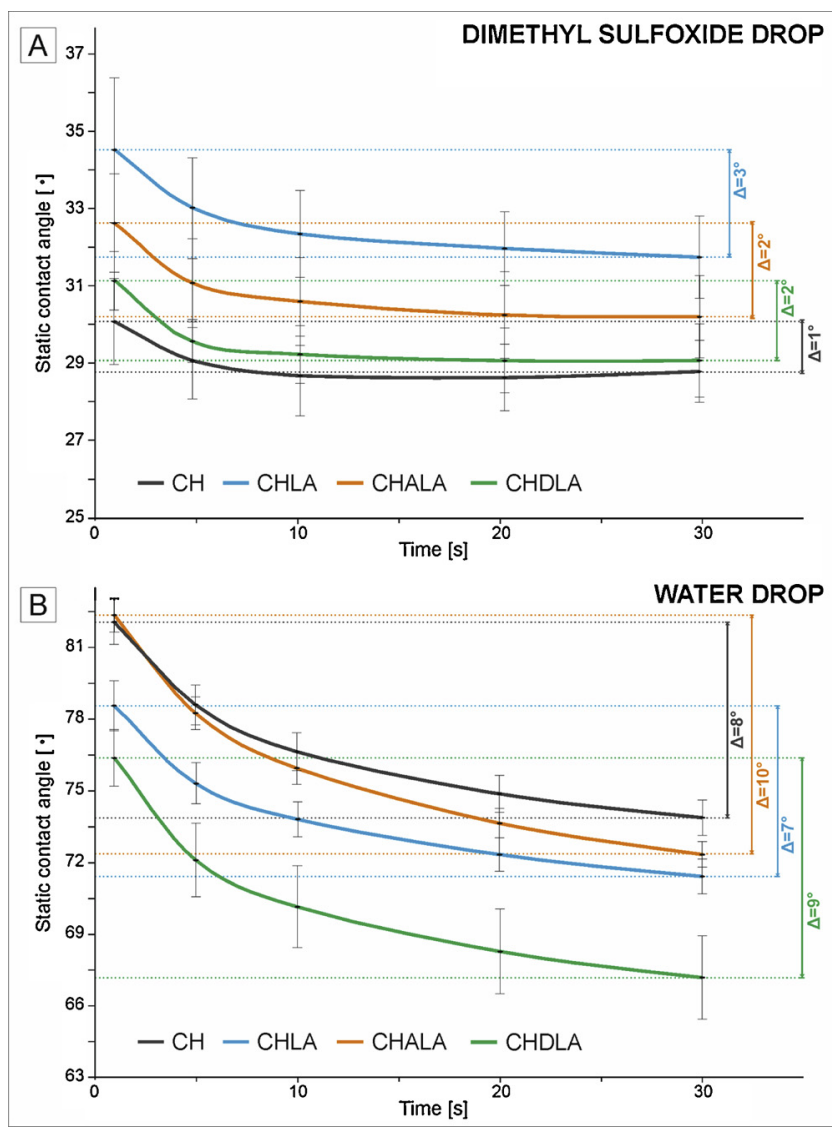

Fig. 7. Static DMSO (A) or water (B) contact angle of the chitosan and CHFA derivatives coatings over $30 \mathrm{~s}$. $\Delta$ represents the difference between the first and the last value.

chains (mainly its amino and hydroxyl groups), while repelling long, hydrophobic FA chains to the outer part of the coating. This effect has been previously observed for $N$-hexanoyl chitosan films: the surface was rich with FA chains (Lee, Ha, \& Park, 1995). Here, our XPS results confirm this: less nitrogen was found in the outermost layer for the CHALA coating, which has a higher substitution degree compared to CHLA (more FA that can organize towards upper layer) and FA chains with much higher mobility, compared to the DLA chains of CHDLA derivative. Further, these results are consistent with our earlier studies into coating organization of chitosan-fatty acids compounds on the surface of two different polyamide substrates: the same chitosan derivative applied as coating can yield two different contact angle values, depending on the character of the coated substrate (Niemczyk, El Fray et al., 2015).

In terms of potential biomedical applications, one of the most important physical parameters of the surface and the coating is wettability, commonly determined by contact angle measurements. However, for a heterogeneous surface such as an amphiphilic coating, the evaluation of the contact angle is complex. The measurement liquid can induce reorganization of the amphiphilic chitosan or chitosan derivative macromolecules. Therefore, the presence of the solvent drop on the coating can change its character, altering the contact angle value. Nevertheless, the analysis of the evolution of the droplet profile in time can be appropriate to determine the wettability of the surface and the self-assembling properties of the coating (Farris et al., 2011). Fig. 7A presents the static water contact angle obtained for the chitosan and CHFA derivatives coatings over a period of $30 \mathrm{~s}$.

The $\theta_{\text {water }}$ of the PET-DLA surface is approx. $95^{\circ}$. For each coating, a lower $\theta_{\text {water value was obtained, indicating that the coating process has }}$ improved the wettability of the surface, resulting in a more hydrophilic character of the surface. Interestingly, the CHFA derivatives exhibit even lower $\theta_{\text {water }}$ values, indicating greater wettability, as compared to unmodified chitosan. The $\theta_{\text {water }}$ values obtained after $30 \mathrm{~s}$ of measurements were as fallows: chitosan $73.9^{\circ} \pm 0.7$, CHALA $72.4^{\circ} \pm 0.5$, CHLA

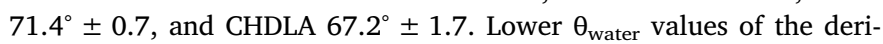
vatives may seem surprising, considering the presence of hydrophobic FA moieties within their structure, however, the presence of the water drop on the coating surface can cause the reorganization of the macromolecules in order to minimize the surface tension of the droplet. Subsequently, the chitosan chains, the hydrophilic part of the amphiphilic derivative, can reorganize toward the surface in contact with water, reducing surface tension and contact angle value. Further, the simultaneous absorption of water by the coating can intensify the molecular interplay. Therefore, the static contact angle measurements of $\mathrm{CH}$ and CHFA derivatives do not reflect the typical surface characteristics described by the Young equation, but rather the changes induced by the interactions with liquid. Here, we would like to emphasize that depending on the thickness of the chitosan/chitosan derivative coating, water absorption, spreading, swelling, and reorganization can take place. Therefore, the interpretation of the static water contact angle needs to be performed with careful analysis of the changes over time (Carneiro-da-Cunha et al., 2010; Farris et al., 2011). Among the CHFA derivatives, the decrease in $\theta_{\text {water }}$ after $30 \mathrm{~s}(\Delta)$ varied, due to structural differences between the FA, the substitution degree, and also different water absorption characteristic of the coatings. The largest decrease in $\theta_{\text {water }}$ was observed for the CHALA coating $\left(\Delta=10^{\circ}\right)$, suggesting that this derivative has the strongest tendency for reorganization.

To further investigate the self-assembly capabilities of the CHFA coatings, the static contact angle studies were repeated using DMSO $\left(\theta_{\text {DMSO }}\right)$. DMSO was chosen for the measurements based on its greater dispersive surface tension component (dispersive to polar ratio of $\sim 4.5$ vs. 0.43 for water), which is a good match for long-chain fatty acids, limited tendency to dissolve the derivatives (reduced solvent absorption), and low volatility, minimizing any change of the drop shape caused by solvent evaporation. Fig. 7B presents the change of $\theta_{\text {DMSO }}$ for the $\mathrm{CH}$ and CHFA derivative coatings over the course of $30 \mathrm{~s}$.

The $\theta_{\text {DMSO }}$ values of all of the coatings are lower than that for uncoated PET-DLA surface, which had $\theta_{\text {DMSO }} \sim 58^{\circ}$, indicating improved wetting of the surface, and the trend is similar to that observed for water. Importantly, we observe a much lower $\theta_{\text {DMso }}$ than $\theta_{\text {water }}$, confirming the presence of dispersive rather than polar groups on the surface, which have a higher affinity for the solvent with the greater dispersive surface tension component. These results are consistent with the XPS analysis, which revealed a greater amount of nitrogen deeper within the coatings, indicating that the FA molecules were at the surface. As a result, the decrease in $\theta_{\text {DMSO }}$ over time is also less pronounced than that observed for water: the $\Delta$ for DMSO on the CHFA coatings is approx. $2-3^{\circ}$ (standard deviation of the measurement is $\leq 1^{\circ}$ ). This less 
pronounced contact angle change may also arise from very low DMSO absorption limiting the mobility of the macromolecules, which is crucial for molecular reorganization.

In order to further examine the interactions of the obtained CHFA coatings with water, we performed dynamic water contact angle measurements. Importantly, the advancing contact angle, formed by the expanding liquid, is a good approximation of Young contact angle for ideal solid surface. As the drop volume increases, the three phase contact point will always encounter a pristine surface, thus diminishing the influence of water absorption and any macromolecular reorganization, as well as averaging out any other effects due to a not perfectly homogenous surface. The results are summarized in Table S4 (SI).

As can be expected, $\theta_{\mathrm{a}}$ values are larger than the static $\theta_{\text {water }}$ values and the differences in $\theta_{\mathrm{a}}$ between unmodified chitosan and the CHFA derivatives are larger than the differences observed in static $\theta_{\text {water }}$ values. Further, the order of the contact angle values, from the lowest to the highest: CHLA, CHALA and CHDLA, is reversed compared to the static measurements results. These results are consistent with the $\theta_{\text {DMso }}$ values and indicate increased hydrophobization of the derivatives with increasing degree of FA substitution, as determined by FTIR analysis. Additionally, using the advancing water contact angle values as an approximate of the Young water contact angle, combined with the DMSO static contact angle (stable after $5 \mathrm{~s}$ ), we were able to estimate the surface fr,ee energy (SFE) of the coatings using the Owens-Wendt method (Owens \& Wendt, 1969) which divides SFE into dispersive and polar components. The results are presented in Table S4. The values calculated for the $\mathrm{CH}$ coating are consistent with those previously described in the literature (Chrzanowska, Gierszewska, Kujawa, Raszkowska-Kaczor, \& Kujawski, 2018; Cunha et al., 2008). Meanwhile, for the CHFA coatings, we observe a higher polar component than for neat chitosan. This is an indicator of the role of the incorporation of the fatty acid on the self-organization of the macromolecules, revealing the expected polar character of chitosan that is normally diminished by inter- and intramolecular interactions and possible trace nonpolar impurities (Cunha et al., 2008). Further, with increasing amount of fatty acid incorporation, the polar character is diminished, due to the dispersive nature of fatty acids. Thus, the SFE analysis is fully consistent with the FTIR and XPS results.

Finally, the receding contact angle is strongly influenced by liquid sorption, retention, and coating swelling; therefore, for most polymers the receding contact angle changes over time. Additionally, any macromolecular mobility of the surface strongly affects the $\theta_{\mathrm{r}}$ value. Consequently, for chitosan and CHFA derivative coatings we were not able to obtain reproducable $\theta_{\mathrm{r}}$ values, meeting measurement quality criteria, and thus we were not able to assess contact angle hysteresis.

Wettability can be correlated with another very important feature of coatings: their friction behaviour and lubricity. In order to determine the coefficient of friction $(\mu)$ and the lubricity of the chitosan and CHFA coatings, hemispherical-shaped probes (Fig. S1 B) were coated and examined in a previously developed biotribological test system (Niemczyk, El Fray et al., 2015) that mimics the conditions of an endovascular catheterization procedure. Fig. 8 presents the evolution of $\mu$ over time for uncoated and coated PET-DLA substrates.

Initially, for all tested materials an increase in the $\mu$ is observed. This is likely the result of wetting-related processes on the dry surface of materials, as all measurements were performed in a liquid mimicking the viscosity of body fluids. However, after $100-200 \mathrm{~s}$, all of the $\mu$ values start to decrease, reaching equilibrium after approx. 1000s. In general, the presence of chitosan or CHFA coatings reduced $\mu$, as compared to neat PET-DLA-as was predicted and is in-line with the contact angle results. The average coefficient of friction of the PET-DLA surface, at equilibrium, was $0.66 \pm 0.24$. For the chitosan coating, the coefficient was reduced to $0.53 \pm 0.36$, while the values of $\mu$ for CHFA derivatives coatings were $0.35 \pm 0.08,0.39 \pm 0.25$, and $0.48 \pm 0.18$ for CHLA, CHALA, and CHDLA, respectively. The $47 \%$ and $41 \%$ reduction in $\mu$ (CHLA and CHALA coating, respectively) indicates improved lubricity of the surface and is a very important advantage of these coatings, in terms of their possible use as coatings for catheters. Further, while we did not conduct a wear analysis, the fact that we do not observe any increase in the coefficient of friction after the initial period suggests that the coatings are stable and sufficiently wear resistant-if the coating was being removed, one would expect the coefficient of frication to increase towards the value of uncoated substrate, or higher. As a result, we conclude that the developed coating process may be able to mitigate the issue of coating delamination or flaking, which have been a recent cause for concern (Chopra et al., 2017; Mehta \& Mehta, 2018).

In order to examine the antimicrobial potential of the coatings, we assessed colonization by model Gram-negative microorganism $E$. coli. Gram-negative microorganisms are responsible for $\sim 30 \%$ of nosocomial infections, with $E$. coli strains among the most common, particularly in the case of bloodstream and urinary tract infections (Peleg \& Hooper, 2010; Sligl, Dragan, \& Smith, 2015). High antibiotic resistance rates limit treatment options, resulting in high mortality rates, thus motivating a focus on prevention, including strategies to reduce adhesion and colonization of device surfaces. Fig. 9 presents the average $E$. coli $\mathrm{CFU}$ per $\mathrm{mm}^{2}$, representing the presence of viable, adherent bacterial cell on the material surface.

Non-parametric Kruskal-Wallis test indicated significant differences between groups (chi-square $=17.1, \mathrm{df}=4$, $\mathrm{p}$-value $=0.002$ ) and nonparametric Conover-Iman post hoc testing identified all but CHDLA as significantly different from PET-DLA substrate $(\mathrm{CH}$ p-value $=0.011$, CHLA p-value $=0.004, \quad$ CHALA p-value $=0.007$, CHDLA $p$ value $=0.400$ ). Therefore we conclude that $\mathrm{CH}$ and both $\mathrm{C} 18$ derivatives (CHLA and CHALA) provide significant, $\sim 80 \%$ reduction in $E$. coli colonization. Only the CHDLA coating did not inhibit $E$. coli colonization. However, this effect may be due to the roughness of this coating (see SEM micrographs in Fig. 5): bacterial adhesion is a complex, multistage process dependent on both surface chemistry, as well as topography (Katsikogianni \& Missirlis, 2004). Additionally, the reduced antimicrobial properties of this coating may also be due to the limited mobility of macromolecules and the lack of free diffusion of covalently bound macromolecules, because our previous work with similar films rather than coatings did not identify this difference (Niemczyk \& El Fray, 2013). A similar effect has been previously described for chemically bound chitosan, which yielded reduced antimicrobial properties, as compared to an ionically bound chitosan coating (Huh et al., 2001). Based on the reduced colonization observed for the CHLA and CHALA coatings and our previous data indicating that similar chitosan-fatty acid derivatives are non-nutritive and can inhibit migration and growth of both Gram-positive and Gram-negative organisms (Niemczyk \& El Fray, 2013), we conclude that the developed coatings may help reduce catheter-associated infections, because both intravascular bloodstream (Edgeworth, 2009) and urinary tract infections (Nicolle, 2014) are primarily caused by microbial colonization outside the body of the patient, followed by migration and growth along the device. However, additional studies examining protein adsorption and biofilm formation will be needed to fully evaluate the antimicrobial potential of the developed coatings.

While an antimicrobial effect was desired, it was important to verify that the coating process, as well as the derivatization of chitosan, did not introduce cytotoxicity towards mammalian cells. Thus, we assessed the cytotoxicity of $\mathrm{CH}$ and CHFA coated discs using a "direct contact" assay, based on the ISO 10993-5 (ISO, 2009). The cytotoxicity tests included using fluorescence microscopy and live/dead staining to assess cell viability, as well as morphological grading of the cellular response (SI Fig. S8). All of the tested materials were determined to be non-cytotoxic, with cell viability exceeding $90 \%$, well beyond the $70 \%$ viability threshold for indicating cytotoxicity provided by the Standard (SI Fig. S9). Likewise, all materials were assessed a morphological reactivity grade of 1 ("slight reactivity"), indicating overall normal 


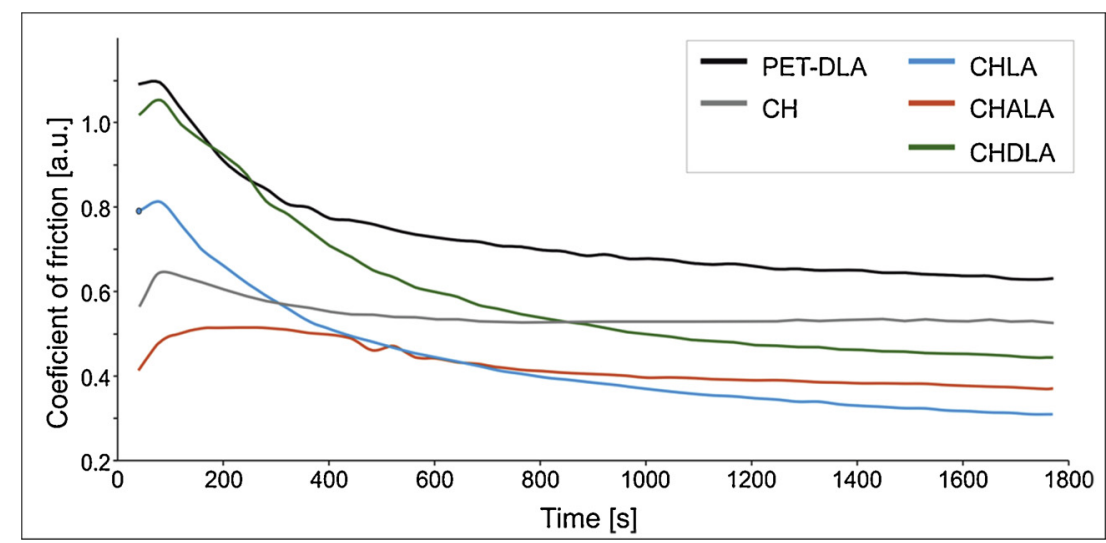

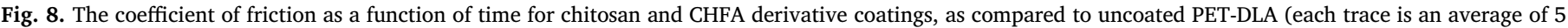
experiments).

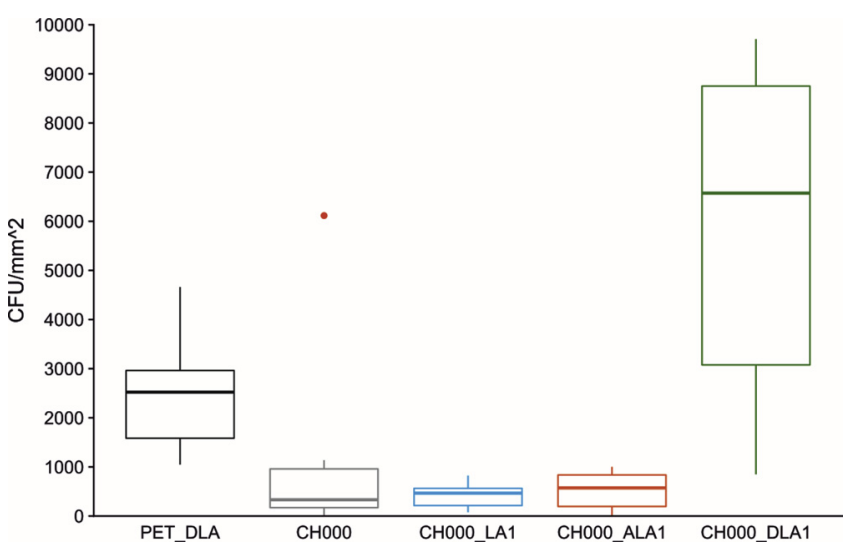

Fig. 9. Box plots presenting bacterial colonization of tested materials following $15 \mathrm{~h}$ of incubation. ( $\mathrm{n}=6$ discs, box represents 1 st and 3rd quartiles, bold bar indicates the median, whiskers represent (at most) 1.5 times the inter-quartile range, red dot indicates potential outlier). (For interpretation of the references to colour in this figure legend, the reader is referred to the web version of this article).

morphology, with only minor morphological changes directly under the disc. We conclude that the developed chemical modification and coating process yields non-toxic coatings-any potentially toxic residual reagents (solvents, coupling agent, etc.) have been adequately removed.

Finally, in order to confirm the potential of the developed coatings to be used for blood-contacting devices, such as endovascular catheters, we also tested the hemocompatibility of the coatings. As part of the study, we placed the tested materials in contact with human whole blood at $37^{\circ} \mathrm{C}$ for $24 \mathrm{~h}$ and compared the red blood cell count (RBC), hemoglobin concentration (HGB), and the mean corpuscular volume (MCV) parameters to blood without contact with the tested materials in the same experimental conditions (negative control), as well as blood not subjected to the conditions of the experiment (blank test). The red blood cell count, total hemoglobin concentration in blood cells, and mean red blood cell volume parameters obtained for all of the tested materials were similar to that of negative control-all were within the range of reference values. To assess hemolytic potential of the coatings, we also measured the free hemoglobin concentration in plasma (fHGB) following 24 -h contact with the tested materials. Uncoated PET-DLA and the $\mathrm{CH}$ coating resulted in values at the level of the negative control $(0.52 \mathrm{~g} / \mathrm{L})$, while the value for CHALA was slightly higher approx. $0.6 \mathrm{~g} / \mathrm{L}$, as compared to $1.0-1.1 \mathrm{~g} / \mathrm{L}$ for CHLA and CHDLA coatings. From these values, we calculated the hemolytic index (HI) for of all of the tested materials, which is presented in Fig. 10.
Despite some differences between the tested materials, the hemolytic index for all of the materials does not exceed $2 \%$ and thus, according to the ASTM F 756-00 standard (ASTM, 2000), chitosan and all of the CHFA derivative coatings can be classified as "non-hemolytic."

\section{Conclusions}

We have described a simple, but effective coating strategy to improve the lubricity and antimicrobial properties of a polymeric surface by covalent coupling of chitosan-fatty acid derivatives. These coating materials were produced by simultaneous $\mathrm{N}, \mathrm{O}$-acylation using carbodiimide coupling agent in acidic environment, thus promoting partial protonation of amino groups and allowing the less reactive primary hydroxyl groups of chitosan to react with fatty acids. The partial protonation also served to retain some amino groups in derivative structure, while simultaneously allowing to avoid commonly applied additional stages of blocking and unblocking reactions. After plasma activation of the polyester substrate, chitosan-fatty acid derivatives were successfully applied as coatings via dip-coating technique. Importantly, XPS depth-profiling elemental analysis and contact angle studies confirmed the self-organization of CHFA biomacromolecules, with the long, hydrophobic FA chains arranging towards the outer part of the coating, and their ability to reorganize. Thus, the coatings resulted in reduced surface roughness and improved wettability. Tribological testing aimed at mimicking a catheter procedure demonstrated that the coatings are lubricious, particularly those with greater chain mobility (CHLA and CHALA). Likewise, for all but the DLA derivative, we observed a significant, $\sim 80 \%$ reduction in bacterial colonization. Finally, cytotoxicity and hemocompatibility studies showed that all of the chitosan-fatty acid derivative coatings are non-toxic and non-hemolytic. We conclude that the developed process and obtained chitosan-fatty acid coatings are promising candidates as a new class of lubricious and antimicrobial coating materials for catheters and other medical devices.

\section{Author contributions}

The manuscript was written through contributions of all authors. All authors have given approval to the final version of the manuscript.

\section{Funding sources}

The authors would like to thank the National Science Centre, Poland (Grant no: N N507 319440 and No 2597/7.PR/2012/2) for providing financial support to this work.

The authors would like to thank prof. Jolanta Baranowska from the Materials Science Institute of The West Pomeranian University of 


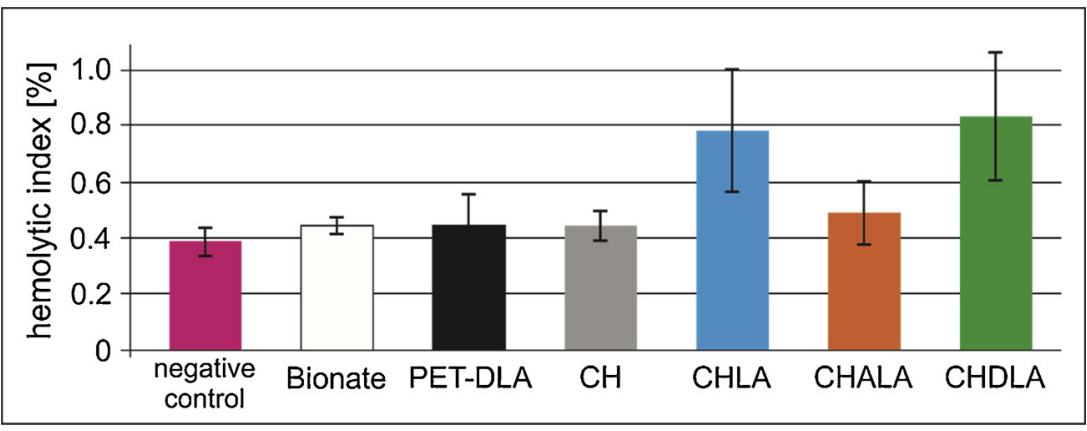

Fig. 10. Hemolytic index of uncoated PET-DLA surface and the CHFA coatings, as compared to the negative control and reference material.

Technology, Szczecin, for discussion on tribological properties.

\section{Appendix A. Supplementary data}

Supplementary data associated with this article can be found, in the online version, at https://doi.org/10.1016/j.carbpol.2019.115263.

\section{References}

Anastasiadis, S. H., Retsos, H., Pispas, S., Hadjichristidis, N., \& Neophytides, S. (2003) Smart polymer surfaces. Macromolecules, 36(6), 1994-1999. https://doi.org/10. $1021 / \mathrm{ma0211129.}$

Aranaz, I., Harris, R., \& Heras, A. (2010). Chitosan amphiphilic derivatives. chemistry and applications. Current Organic Chemistry, 14(3), 308-330. https://doi.org/10.2174/ 138527210790231919.

ASTM (2000). Standard practice for assessment of hemolytic properties of materials. ASTM F756-OOhttps://doi.org/10.1520/F0756-00.

Baum, R. A., \& Baum, S. (2014). Interventional radiology: A half century of innovation. Radiology, 273(Suppl. 2), S75-91. https://doi.org/10.1148/radiol.14140534.

Canfield, J., \& Totary-Jain, H. (2018). 40 years of percutaneous coronary intervention: History and future directions. Journal of Personalized Medicine, 8(4), 33. https://doi. org/10.3390/jpm8040033.

Carneiro-da-Cunha, M. G., Cerqueira, M., Souza, B. W. S., Carvalho, S., Quintas, M., Teixeira, J., \& Vicente, A. (2010). Physical and thermal properties of a chitosan/ alginate nanolayered PET film. Carbohydrate Polymers, 82(1), 153-159. https://doi. org/10.1016/j.carbpol.2010.04.043.

Casey, A. L., Mermel, L. A., Nightingale, P., \& Elliott, T. S. (2008). Antimicrobial central venous catheters in adults: A systematic review and meta-analysis. The Lancet Infectious Diseases, 8(12), 763-776. https://doi.org/10.1016/S1473-3099(08) 70280-9.

Chopra, A. M., Mehta, M., Bismuth, J., Shapiro, M., Fishbein, M. C., Bridges, A. G., \& Vinters, H. V. (2017). Polymer coating embolism from intravascular medical devices - A clinical literature review. September Cardiovascular Pathology, 30, 45-54. https://doi.org/10.1016/j.carpath.2017.06.004.

Chrzanowska, E., Gierszewska, M., Kujawa, J., Raszkowska-Kaczor, A., \& Kujawski, W. (2018). Development and characterization of polyamide-supported chitosan nanocomposite membranes for hydrophilic pervaporation. Polymers, 10(8), 868. https:// doi.org/10.3390/polym10080868.

Croisier, F., \& Jérôme, C. (2013). Chitosan-based biomaterials for tissue engineering. European Polymer Journal, 49(4), 780-792. https://doi.org/10.1016/j.eurpolymj. 2012.12.009.

Cunha, A. G., Fernandes, S. C. M., Freire, C. S. R., Silvestre, A. J. D., Neto, C. P., \& Gandini, A. (2008). What is the real value of chitosan's surface energy? Biomacromolecules, 9(2), 610-614. https://doi.org/10.1021/bm701199g.

Dambies, L., Guimon, C., Yiacoumi, S., \& Guibal, E. (2001). Characterization of metal ion interactions with chitosan by X-ray photoelectron spectroscopy. Colloids and Surfaces A: Physicochemical and Engineering Aspects, 177(2-3), 203-214. https://doi.org/10. 1016/S0927-7757(00)00678-6.

Dellimore, K. H., Franklin, S. E., \& Helyer, A. R. (2014). A review of catheter related complications during minimally invasive transcatheter cardiovascular intervention with implications for catheter design. Cardiovascular Engineering and Technology, 5(3), 217-232. https://doi.org/10.1007/s13239-014-0183-9.

Edgeworth, J. (2009). Intravascular catheter infections. The Journal of Hospital Infection, 73(4), 323-330. https://doi.org/10.1016/J.JHIN.2009.05.008.

El Fray, M., \& Czugala, M. (2012). Polish artificial heart program. Wiley Interdisciplinary Reviews: Nanomedicine and Nanobiotechnology, 4(3), 322-328. https://doi.org/10. 1002/wnan.175.

Farris, S., Introzzi, L., Biagioni, P., Holz, T., Schiraldi, A., \& Piergiovanni, L. (2011). Wetting of biopolymer coatings: Contact angle kinetics and image analysis investigation. Langmuir, 27(12), 7563-7574. https://doi.org/10.1021/la2017006.

Feneley, R. C. L., Hopley, I. B., \& Wells, P. N. T. (2015). Urinary catheters: History, current status, adverse events and research agenda. Journal of Medical Engineering \& Technology, 39(8), 459-470. https://doi.org/10.3109/03091902.2015.1085600.

Gilchrist, I. C., \& Kozak, M. (2010). Hydrophilic-coated radial sheaths: A leap forward, but watch where you land. JACC Cardiovascular Interventions, 3(5), 484-485. https:// doi.org/10.1016/j.jcin.2010.03.008.

Huh, M. W., Kang, I.-K., Lee, D. H., Kim, W. S., Lee, D. H., Park, L. S., ... Seo, K. H. (2001). Surface characterization and antibacterial activity of chitosan-grafted poly(ethylene terephthalate) prepared by plasma glow discharge. Journal of Applied Polymer Science, 81(11), 2769-2778. https://doi.org/10.1002/app.1723.

Ikada, Y. (1994). Surface modification of polymers for medical applications. Biomaterials, 15(10), 725-736. https://doi.org/10.1016/0142-9612(94)90025-6.

ISO (2009). Biological evaluation of medical devices-Part 5: Tests for in vitro cytotoxicity. ISO 10993-5:2009 Geneva, Switzerland.

Katsikogianni, M., \& Missirlis, Y. (2004). Concise review of mechanisms of bacterial adhesion to biomaterials and of techniques used in estimating bacteria-material interactions. European Cells and Materials, 8, 37-57. https://doi.org/10.22203/eCM. v008a05.

Kurita, K., Ikeda, H., Shimojoh, M., \& Yang, J. (2007). N-phthaloylated chitosan as an essential precursor for controlled chemical modifications of chitosan: Synthesis and evaluation. Polymer Journal, 39(9), 945-952. https://doi.org/10.1295/polymj. PJ2007032.

Lee, K. Y., Ha, W. S., \& Park, W. H. (1995). Blood compatibility and biodegradability of partially N-acylated chitosan derivatives. Biomaterials, 16(16), 1211-1216.

Lee, S. D., Hsiue, G. H., \& Kao, C. Y. (1996). Preparation and characterization of a homobifunctional silicone rubber membrane grafted with acrylic acid via plasmainduced graft copolymerization. Journal of Polymer Science, Part A: Polymer Chemistry, 34(1), 141-148. https://doi.org/10.1002/(SICI)1099-0518(19960115) 34:1<141::AID-POLA15 > 3.0.CO;2-L.

Lin, D. M.-H., \& Wu, Y. (2018). Implantable vascular access devices - past, present, and future. Transfusion, 58, 545-548. https://doi.org/10.1111/trf.14485.

Mehta, R. I., \& Mehta, R. I. (2018). Hydrophilic polymer embolism: Implications for manufacturing, regulation, and postmarket surveillance of coated intravascular medical devices. Journal of Patient Safety, 1-11. https://doi.org/10.1097/pts. $000000000000047300(00)$

Nacey, J. N., \& Delahunt, B. (1991). Toxicity study of first and second generation hydrogel-coated latex urinary catheters. British Journal of Urology, 67(3), 314-316. https://doi.org/10.1111/j.1464-410X.1991.tb15143.x.

Nicolle, L. E. (2014). Catheter associated urinary tract infections. Antimicrobial Resistance and Infection Control, 3, 23. https://doi.org/10.1186/2047-2994-3-23.

Niemczyk, A., \& El Fray, M. (2013). Novel chitosan derivatives as films with an antimicrobial effect. Progress on Chemistry and Application of Chitin and Its Derivatives, XVIII, 59-66.

Niemczyk, A., El Fray, M., \& Franklin, S. E. (2015). Friction behaviour of hydrophilic lubricious coatings for medical device applications. Tribology International, 89, 54-61. https://doi.org/10.1016/j.triboint.2015.02.003.

Niemczyk, A., Kaczorowski, P., \& El Fray, M. (2015). Spin-coated chitosan on copolyester substrates. Progress on Chemistry and Application of Chitin and Its Derivatives, $X X$, 236-245. https://doi.org/10.15259/PCACD.20.23.

Niemczyk, A., Kmieciak, A., El Fray, M., \& Piegat, A. (2016). The influence of C18-fatty acids on chemical structure of chitosan derivatives and their thermal properties. Progress on Chemistry and Application of Chitin and Its Derivatives, 21, 165-175. https://doi.org/10.15259/PCACD.21.18.

Oliveira, J. R., Martins, M. C. L., Mafra, L., \& Gomes, P. (2012). Synthesis of an O-alkynylchitosan and its chemoselective conjugation with a PEG-like amino-azide through click chemistry. Carbohydrate Polymers, 87(1), 240-249. https://doi.org/10.1016/j. carbpol.2011.07.043.

Owens, D. K., \& Wendt, R. C. (1969). Estimation of the surface free energy of polymers. Journal of Applied Polymer Science, 13(8), 1741-1747. https://doi.org/10.1002/app. 1969.070130815.

Parada, G. A., Yuk, H., Liu, X., Hsieh, A. J., \& Zhao, X. (2017). Impermeable robust hydrogels via hybrid lamination. Advanced Healthcare Materials, 6(19), 1700520. https://doi.org/10.1002/adhm.201700520.

Peleg, A. Y., \& Hooper, D. C. (2010). Hospital-acquired infections due to gram-negative bacteria. The New England Journal of Medicine, 362(19), 1804-1813. https://doi.org/ 10.1056/NEJMra0904124.

Piegat, A., \& El Fray, M. (2007). Polyethylene terephthalate modification with the monomer from renewable resources. Polimery, 52(11), 885-888.

Singha, P., Locklin, J., \& Handa, H. (2017). A review of the recent advances in antimicrobial coatings for urinary catheters. Acta Biomaterialia, 50, 20-40. https://doi. org/10.1016/j.actbio.2016.11.070. 
Sligl, W. I., Dragan, T., \& Smith, S. W. (2015). Nosocomial Gram-negative bacteremia in intensive care: Epidemiology, antimicrobial susceptibilities, and outcomes. International Journal of Infectious Diseases, 37, 129-134. https://doi.org/10.1016/J. IJID. 2015.06.024

Sobolewski, P., \& El Fray, M. (2015). Cardiac catheterization: Consequences for the endothelium and potential for nanomedicine. Wiley Interdisciplinary Reviews:

Nanomedicine and Nanobiotechnology, 7(3), 458-473. https://doi.org/10.1002/wnan. 1316.

Staniszewski, Z., Piegat, A., Okroj, W., Walkowiak-Przybylo, M., Jakubowski, W.,

Walkowiak, B., \& El Fray, M. (2017). The effect of carbon nanoparticles on biological properties of polyester nanocomposites. Journal of Biomaterials Applications, 31(10), 1328-1336. https://doi.org/10.1177/0885328217706193.

Staniszewski, Z., Sobolewski, P., Piegat, A., \& El Fray, M. (2018). The effects of nano-sized carbon fillers on the physico-chemical, mechanical, and biological properties of polyester nanocomposites. European Polymer Journal, 107, 189-201. https://doi.org/ 10.26434/chemrxiv.6300095.

Theapsak, S., Watthanaphanit, A., \& Rujiravanit, R. (2012). Preparation of chitosancoated polyethylene packaging films by DBD plasma treatment. ACS Applied Materials \& Interfaces, 4(5), 2474-2482. https://doi.org/10.1021/am300168a.

Wu, D. H., Chen, A. D., \& Johnson, C. S. (1995). An improved diffusion-ordered spectroscopy experiment incorporating bipolar-gradient pulses. Journal of Magnetic Resonance Series A, 115(2), 260-264. https://doi.org/10.1006/JMRA.1995.1176.

Wyman, P. (2012). Hydrophilic coatings for biomedical applications in and ex vivo. Coatings for biomedical applications3-42. https://doi.org/10.1016/B978-1-84569-568-2. 50001-1. 